\title{
Theoretical Underpinnings of eHealth Impact Assessment for Healthcare Service Delivery: Systematic Review
}

\author{
Amuomo Nixon', Ondulo Jasper ${ }^{2}$, Wagacha Peter $^{3}$ \\ Department of Information Technology, School of Computing and Informatics, \\ Masinde Muliro University of Science and Technology P.O. Box, 190 - 50100, Kakamega, Kenya ${ }^{1,2}$ \\ School of Computing and Informatics, University of Nairobi P.O. Box, 30197-00100, Nairobi, Kenya ${ }^{3}$
}

\begin{abstract}
While it is true that ICT has a positive impact on health service delivery, the United Nations agencies and global health authorities, point out challenges with eHealth implementations and impact evidence. These are often poorly reported thus limiting stakeholder's visibility of the competing investment initiatives. Using theory as a 'lens' for studying eHealth impact assessment with the emphasis on healthcare service delivery, provides a roadmap for the phenomenon key factors and their interactions. The use of theories, helps in explaining eHealth adoption, implementation processes and their consequent influences on the organization, social and the environment. The paper reviewed related theories and research specific to the field of eHealth impact assessment through multilevel approach at the organization level. The review was conducted based on the Preferred Reporting Items for Systematic Reviews and Meta-Analyses (PRISMA) and also on the Population, Intervention, Comparison, Outcome, and Study Design (PICOS) method. In conclusion, the resultant eHealth impact assessment incorporated various components from different theories, models and frameworks to underpin the outcome framework.
\end{abstract}

Keywords: eHealth, eHealth impact, impact assessment, healthcare service delivery, theory

\section{I.BACKGROUND}

Cambridge University Press [1] dictionary, defines 'Theory' as; 'a formal statement of the rules on which a subject of study is based or of ideas that are suggested to explain a fact or event or, more generally, an opinion or explanation'. On the other hand, Encyclopedia Britannica [2] defines scientific theory as a systematic ideational structure of broad scope, conceived by the human imagination, that encompasses a family of experiential laws in a structure that scientifically explain them in a rational manner. According to [3], most scientific theories contain certain hypotheses whose nature is arbitrary, making them mere speculative constructions or free inventions of the human mind. [4], are of the view that scientists formulate theories, test, accept, reject, modify, and use theories as guides to understanding and predicting events in the world about them. [5], on the other hand argues that no theory with constructive elements in its base can be final, there is always a chance for future measurements disproving its predictions. These views, therefore formed the basis of this study.

Thus, in theoretical underpinning we, looked at what has been done in related fields, applications and approaches, with a view to either using the same or modifying them appropriately to guide eHealth implementation assessment. In this study, the researchers agrees with [5], notably, by scrutinizing some of the earlier developed theories, models and frameworks as they focused on eHealth adoption and implementation. A process which therefore guided, the theoretical underpinnings for eHealth impact assessments. This was further seen to be critical given that stakeholders in healthcare are seeking ways to address factors of health, given its complexity and more so, it concerns everyone. Ill health handicaps people, economies and development, thus raising the need to address its negative effects by applying Universal Health Coverage concept by the use of ICT, which has been regarded as an enabler in diverse domains or fields. The need notwithstanding, there seems to be no uniform approach to deliver a successful eHealth (loosely regarded as leveraging ICT in health) implementation to would be beneficiaries. Further, there is lack of an agreed eHealth impact assessment framework that can be used to show evidence of implementation benefits to the beneficiaries and return of investment (ROI). 
According to [6], eHealth interventions typically addresses people needs, user satisfaction and an evaluation on their effectiveness, an assessment of their attributable impact and the value for money for such interventions. Thus it is arguably reasonable to assess, verify and hence justify its adoption and implementation. Notably, attention has been given to eHealth benefits identification and its impact to the patients and healthcare providers given that it has become a meeting point between health informatics, public health and the business.

The use of eHealth has been envisaged to provide several benefits such as informed patients and caregivers. With direct access to data, information and knowledge about health issues and their impact on wellness and health facilities, patients are therefore able to make effective decisions about their health and lifestyles. With the information designed around the patient; healthcare providers can therefore share and have access to more patients for focused attention, thereby increasing the benefits to the patients. The process of eHealth benefit realization starts with access to information and knowledge sharing as shown in figure 1 below.

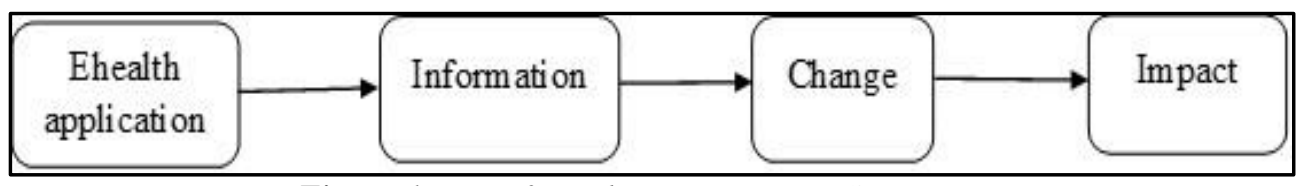

Figure 1: Benefit realization process: Source: [7].

The use of eHealth applications, helps in data gathering, which then leads to information formulation for possible sharing. Data gathering, processing, retrieval and sharing; when well adopted and implemented, has the potential to redefine clinical processes, work practices and workflow in administrative or support to healthcare service delivery. Depending on the execution, a much faster, moderate or slower familiar procedures can then be realized. These changes bring about the benefits seen at the end, such as; patient centeredness, timeliness, safety, access, efficiency and effectiveness; which eventually flows or ends up permeating the environment, organization and the society in diverse ways . This supports [8], assertion that health services should be safe, effective, patient centered, timely, efficient, equitable, acceptable (respectful) and continuity assured.

With the increasing technological advancements, it is inevitable for social and cultural changes in the health sector as it takes into account ICT as one of its way of doing business. It is paramount to have an enabling environment for the organizations to strategically plan for ehealth to success [9]. The combination of the technical capabilities, society and the environment, forms 'ecosystem' for eHealth in a country. The introduction of ICT causes changes to organization structures and therefore, organizations need to formulate strategies on how healthcare services should be developed and deployed to stimulate its benefits. The disruptive digital technologies would therefore impact the environment, organization strategy and the society [10].

The discussion below looked at the models, frameworks and theories which anchored this review. The value chain model shown in figure 2, shows that, the impacts such as institutional changes beyond the target group [11]. The model is divided into four main assessment targets as; readiness, availability, uptake and impact, each with its measurements elements. In this compilation, the key focus is on impacts assessment stage.

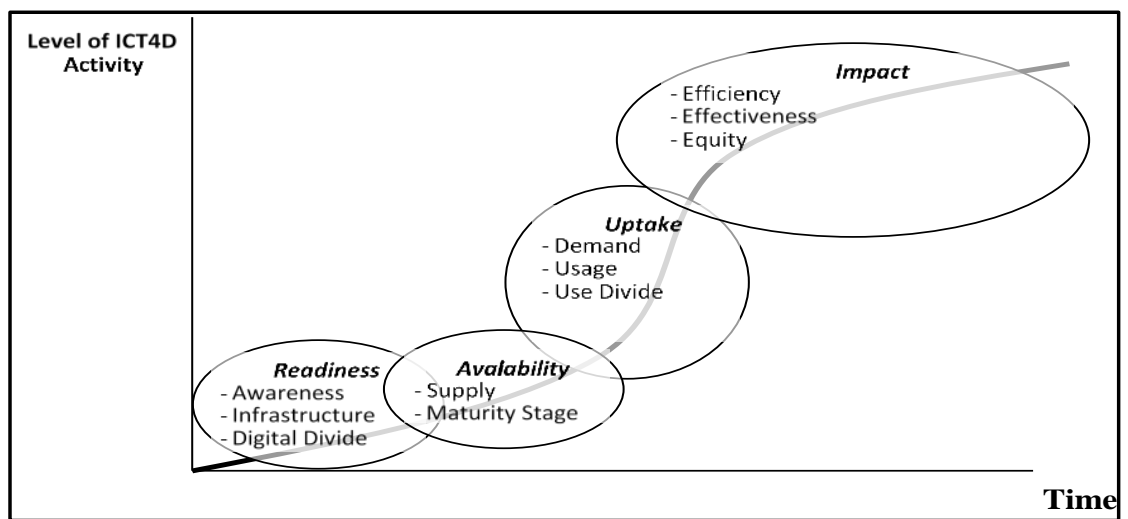

Figure 2: ICT4D Assessment over time refocused. Source: [12] 
The value chain demonstrated that, to understand the impact of ICT4D holistically, the conceptualization must include the dimensions in figure 3, covering the potential impact on social, environment and economic objectives along the intersecting value chains (VC's). The value chain's interacting layers such as divisions, departments, teams, and individuals often have some degree of interdependence that leads to bottom-up and top-down influence mechanisms. The patients and caregivers found at a facility (organization), are contexts for influence on individual cognitions, attitudes, and behaviors [13] top-down effects. Similarly, individual cognitions, attitudes, and behaviors can also influence the functioning and outcomes of the organization [14] bottom-up effects.

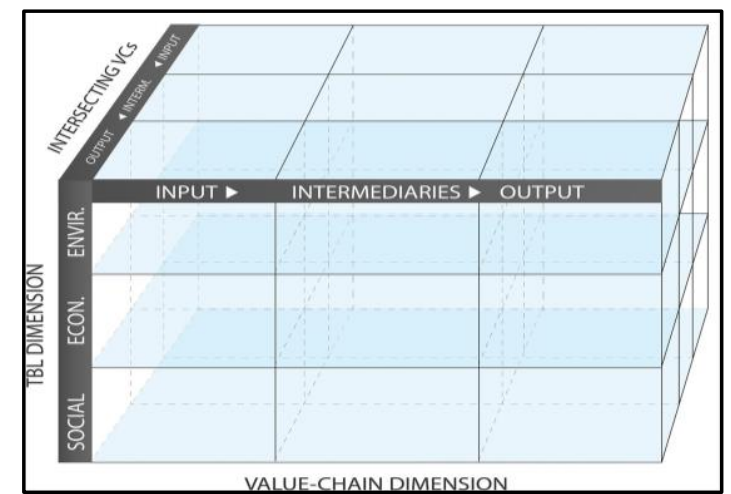

Figure 3: Three dimensions of ICT4D impact areas. Source: [15]

\section{Key concepts underpinning eHealth impact assessment framework}

By using theories, generalizability of the benefits is realized through a framework. The frameworks can then be applied in a different setting and validated to add to the body of knowledge. Today, we have numerous appraisal models used in healthcare organizations to gauge particular aspects in the field and its environs. Different types of eHealth impact assessment models, frameworks, theories were reviewed by the authors and identified gaps highlighted, integrated to formulate a new conceptual framework. The authors marked the gaps by use of an asterix (*) on the missing constructs in Normalization process theory (NPT), Networked readiness index (NRI), Technology organization environment framework (TOE), and the Strategy, Technology, Organization, People, and Environment (STOPE); which we reviewed for the purpose of this study.

\section{Normalization Process Theory (NPT)}

Normalization process theory (NPT) was developed by [16] . The NPT theory uses socio logical theoretical framework in addressing individual and organizational factors. It has the potential to bridging the gap between researchers on eHealth impact assessment models and theoretical underpinnings in line with eHealth implementations. Normalization processes theory aims at addressing how innovations can be operationalized into daily practice for health care delivery [17] and [18]. NPT argues that material practices become routinely embedded in social contexts as a result of people working, individually and collectively to implement them.

NPT is composed of three broad contexts; Social, Organizational and Technical. The authors of this model suggested coherence, cognitive participation, collective action, and reflexive monitoring as a means for successful operationalization of health innovations into routine work ([19]; [16]; [17]). NPT advocates for the involvement of all stakeholders in the implementation processes, takes note of the work they have to do individually and collectively, and the subtle and gradual processes of embedding and integrating to normalization.

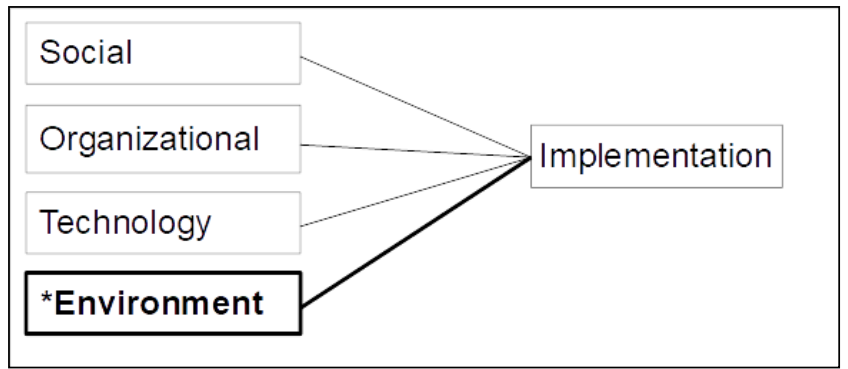

Figure 4: NPT constructs visualization diagram 
From the NPT review, the researchers realized there was a gap on environmental coverage and therefore added the environment as construct marked with an asterix.

\section{Networked Readiness Index (NRI)}

Networked Readiness Index (NRI) is one of the assessment framework that has been used internationally by countries to assess their capacity on ICT use. More than a decade, NRI has provided useful conceptual framework for evaluation of ICT impact, benchmarking ICT readiness and usage of their economies to decision makers globally. It is also the first global framework to be used for mapping out capacity factors. NRI by definition, is the degree to which a community is prepared to participate in the networked world.

Networked Readiness Index (NRI) framework has four sub-indexes used for measurements: (i) the ICT Environment (ii) the Readiness of a society to use ICTs (iii) Actual ICT Usage by all primary stakeholders and (iv) ICT Impacts generation in the economy and society.

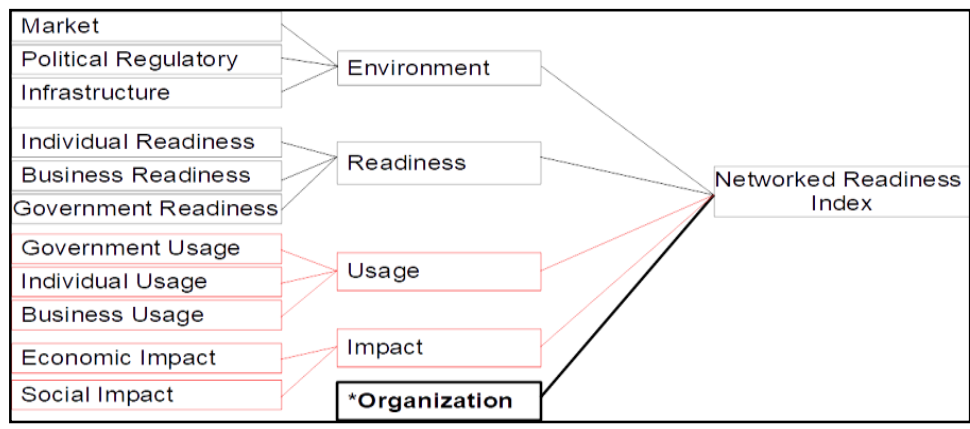

Figure 5: The Networked Readiness Index Framework. Source: [20].

From NRI review, the researchers realized there was a gap on the organization coverage and therefore added the organization as construct marked with an asterix.

\section{Technology Organization Environment (TOE) Framework}

Tornatzky and Fleisher developed the technology organization environment framework, in 1990, which described factors that affect technology adoption and its likelihood of success. The framework, looks at how the technology context, the organizational context, and the environmental context affects implementation [21]. The technological context looks at the internal and external technologies relevant to the firm such as processes, current practices and equipment's availability to the firm internally and externally. The organizational context looks at the characteristics and resources including the firm's size, degree of centralization, degree of formalization, managerial structure, human resources, amount of slack resources, and linkages among employees. While the environmental context, is the space in which a firm conducts its business, competitors, and dealings with the government, the macroeconomic context, and the regulatory environment [21]. The technological, organizational and environment contexts, influence the way a firm evaluates the need for and adopts new technologies.

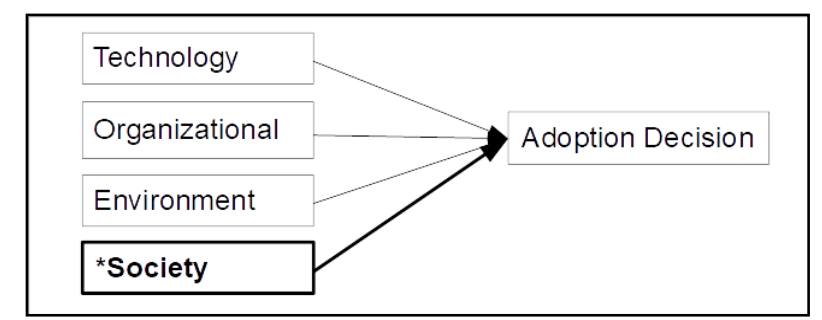

Figure 6: Technology, organization, and environment framework. Source:[21].

From the TOE review, the researchers realized there was a gap on societal coverage and therefore added the society as construct to the framework marked with an asterix. 


\section{The STOPE framework}

The STOPE model, an acronym for Strategy, Technology, Organization, People, and Environment, was developed by [22]. The model has been used for e-government which has similarities with eHealth readiness in other studies. An example, is the study by [23], where STOPE model constructs like technology resources, management system, and government, plus individual commitment in championing technology infrastructure, were reported as the essentials for e-readiness in general. A study by [24], reveals that e-government strategy affects technology and people's readiness to adopt technology. Figure 7 below shows STOPE theoretical framework.

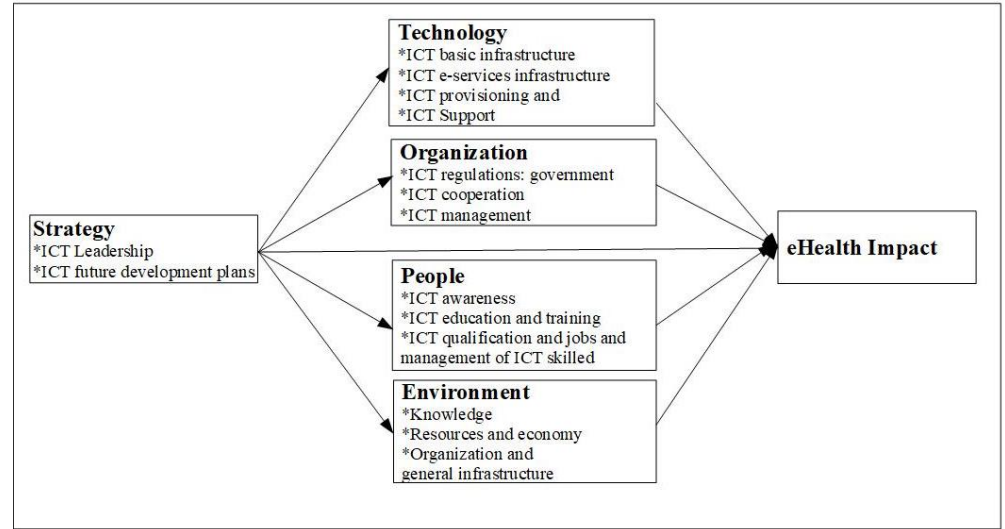

Figure 7: Theoretical Framework - STOPE

The Universal Health Coverage Framework

A Universal Health Coverage study for the western pacific region [6] titled, 'Moving Towards Better Health', identified five attributes for high impact UHC framework as quality, efficiency, equity, access, accountability, and sustainability and resilience. [25], is of the view that health sector has realized a turnaround due to ICT, which has become a cornerstone for efficient and effective healthcare service delivery. ICT has been useful in data gathering, healthcare services administration and the potential for quality and reliability improvement of healthcare services. The impact of ICT tools usage on health information dissemination across geographically and socially isolated areas cannot be overemphasized [26].

TABLE 1: EHEALTH IMPACT ATTRIBUTES SOURCE: LITERATURE

\begin{tabular}{|l|l|}
\hline Attributes & Action domains for UHC \\
\hline Effectiveness & $\begin{array}{l}\text { The ability of eHealth system to improve diagnostic accuracy and treatment, helps in informed } \\
\text { care which is based on evidence, improves communication and information exchange between } \\
\text { patients and service providers, aid in more people-centred care and adherence to treatment. } \\
\text { Maximize resources utilization, avoiding waste and decreasing costs [27]. }\end{array}$ \\
\hline Efficiency & $\begin{array}{l}\text { Resource optimization usage, reduction of fragmentation, reduction in duplication and } \\
\text { unnecessary care, and improvement in efficiency through real time information access. }\end{array}$ \\
\hline Access & $\begin{array}{l}\text { Improvement of access to quality healthcare services for the people and empowering } \\
\text { individuals to make more informed health decisions. Enhanced communication among health } \\
\text { practitioners and patients and data processing at the facilities [28], and elimination of } \\
\text { geographical barriers through efficient consultations amongst medical practitioners and } \\
\text { establishing continuous communication with patients [29]. }\end{array}$ \\
\hline Accountability & $\begin{array}{l}\text { Through information and communication sharing, transparency and accountability is } \\
\text { improved. }\end{array}$ \\
\hline Sustainability & $\begin{array}{l}\text { Strengthening monitoring of public health threats and improvement of overall health system } \\
\text { adaptability. }\end{array}$ \\
\hline Safety & $\begin{array}{l}\text { The use of information systems believed to improve legibility and errors reduction and } \\
\text { boosting the quality of healthcare [30]. The comfort and confidence among patients is } \\
\text { attributed to an enriched social presence from multi physicians attending to the patient. }\end{array}$ \\
\hline The use of ICT saves significant amount of time in coordinating and managing hospital \\
operations seamless for patient centered care management.
\end{tabular}




\section{II.METHODOLOGY}

A systematic review of literature was used to understand the dynamics involved in eHealth impacts assessments using secondary data. The review revealed gaps and similarities which were analyzed as a 'lens' to inform the new amalgamated eHealth impact assessment framework. Systematic literature reviews are at times evaluated for eligibility based on relevance and acceptability [31]. Thus, the narrative review type was used. This type of review is very useful in gathering volumes of literature in a specific subject area and synthesizing it. Its primary goal was to provide a comprehensive background for understanding the current knowledge and to highlight the significance of the new research area.

The researcher adopted search and selection criteria based on Preferred Reporting Items for Systematic Reviews and Meta-Analyses (PRISMA) for relevant abstracts and full text articles with a total of 59 published article. 37 articles were incorporated into this review as per the inclusion and exclusion criteria based on the Population, Intervention, Comparison, Outcome, and Study Design (PICOS). The inclusion criteria included articles covering thematic topics on eHealth implementations, eHealth benefits assessments and health sector as the focus area. The flow diagram in figure 8 , gives a summary of citation retrieval and selection of articles.

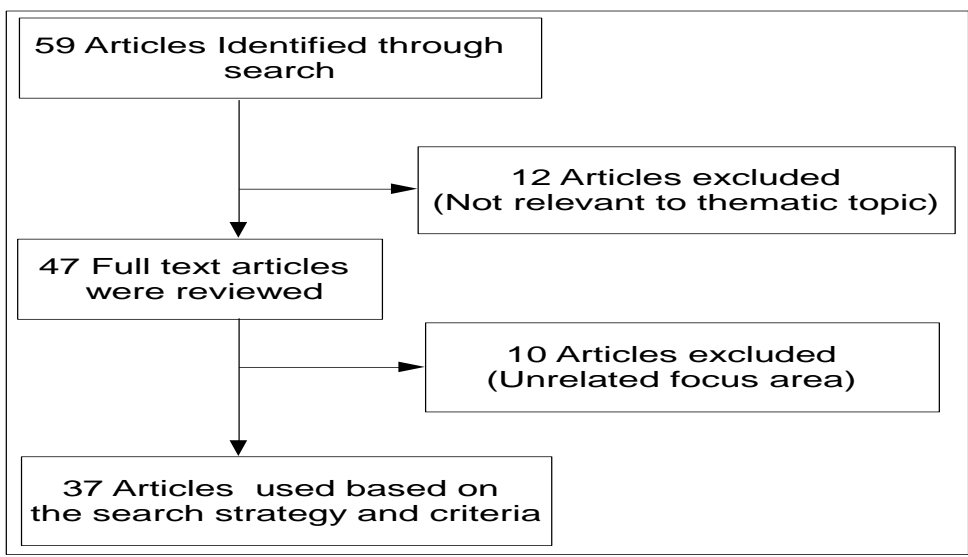

Figure 8: Methodology flowchart used for the selection of articles in the study.

\section{III.RESULTS}

The synthesized theories and frameworks of Normalization Process Theory (NPT), Technology, Organization, and Environment framework (TOE), Networked Readiness Index (NRI) framework, the STOPE framework and the five eHealth Assessment Models, have been used to amalgamate a conceptual framework in figure 10. The conceptual framework incorporated theoretical underpinnings of e-impact factors for a holistic theoretical framework on eHealth. It took into account inclusion of societal factors into TOE, inclusion of environmental construct into NPT, addition of the organization constructs to NRI and incorporation of societal factors into STOPE, as depicted diagrammatically in figure 9.

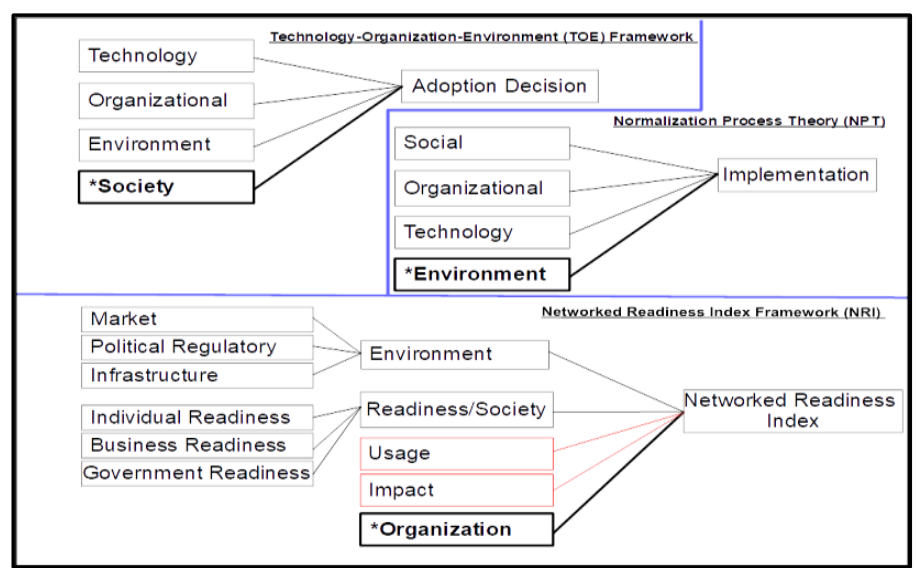

Figure 9: Diagrammatic combination of theories, frameworks and models. 


\title{
International Journal of Advanced Research in Computer and Communication Engineering
}

\author{
Vol. 9, Issue 8, August 2020
}

\section{DOI 10.17148/IJARCCE.2020.9801}

[32], connects theory and practice and contends that former guides the latter. Practice enables testing of a theory while generating research questions. A research therefore, contributes to theory building and selection of practice guidelines. [32] theorize that, careful interweaving of theory and research reinforces learning through practice and creates knowledge fabric for any discipline. They further defined a concept as, a symbolic representation of an abstract idea. [33], described concepts as, the components of theory which convey the abstract ideas within a theory and a complex mental formulation of experience. The systematic theories reviewed formed the basis of generalization of the components to underpin the constructs in an integrated construct relationship diagram as shown in figure 10 by the authors.

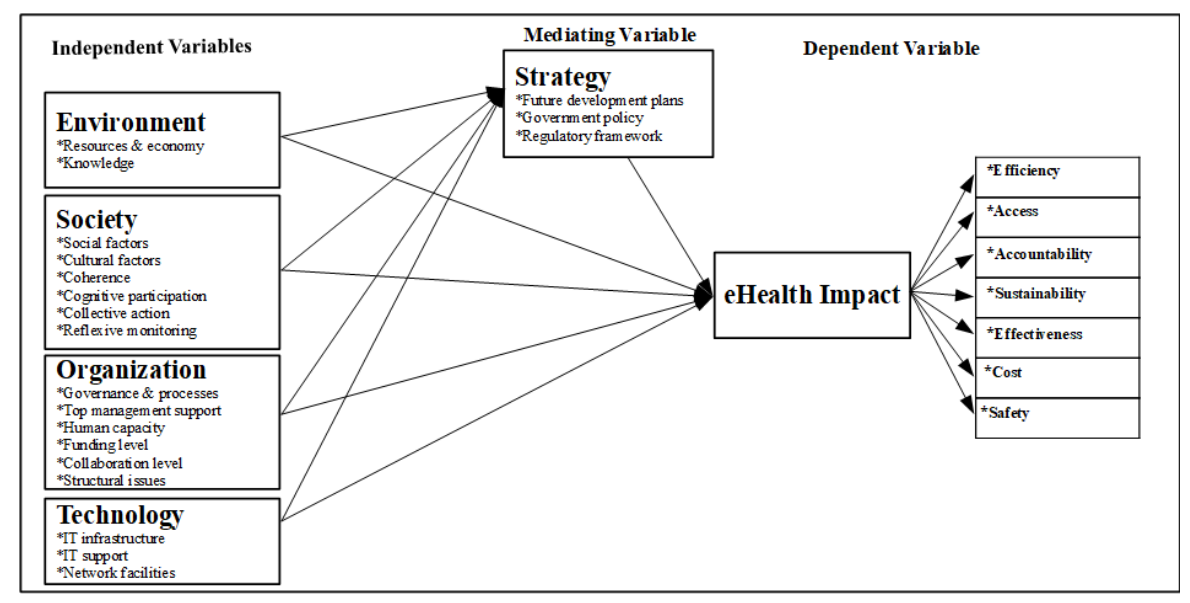

Figure 10: Integrated abstract construct relationship.

\section{IV.CONCLUSION}

The integrated eHealth impact assessment diagram in figure 10, illustrates a combination of constructs from theories and models such as the environment, organization, society and technology. The organization's strategic planning is a key component that moderates the rest of the independent variables to the dependent variable. The study has contributed a new holistic view of the underpinning principles for eHealth impact assessment. The researchers recommend further empirical validation the integrated eHealth assessment framework because previous studies have shown that, despite all the assessments done, there has been no clearly validated eHealth assessment framework for successful implementation of eHealth systems.

\section{REFERENCES}

[1] C. U. Press, "Cambridge University Press," Cambridge University Press 2020 , p. 0, 2020.

[2] E. Britannica, "Encyclopedia Britannica," Encyclopedia Britannica, 2018.

[3] A. Einstein, "The Library of Living Philosopher," In Albert Einstein: Philosopher-Scientist (ed. Schilpp, P. A.), Evanston, Illinois, 1949.

[4] J. \&. J. J. Jaccard, "Theory construction and model-building skills: A practical guide for social scientists.," Guilford Press., 2010.

[5] S. Chatterjee, "The Nature of Scientific Theory.," Current science., Vols. 102. 386-388., 2012.

[6] "Universal Health Coverage: Moving towards better health. Manila (Philippines)," WHO, 2016b. World Health Organization. , vol. WHO Regional Office for the Western Pacific., 2016.

[7] K. \&. J. T. \&. D. A. \&. S. V. Stroetmann, "eHealth is Worth it The economic benefits of implemented eHealth solutions at ten European sites.," no. 10.13140/2.1.1551.9367., 2006.

[8] I. o. M. (IOM), "Crossing the Quality Chasm: A New Health System for the 21st Century," Washi, 2001.

[9] I. WHO, "National eHealth Strategy Toolkit.," 2012.

[10] E. Union, "Assessing the impact of digital transformation of health services.," 2019.

[11] F. \&. E. L. \&. D. M. \&. T. F. Kivunike, "Towards a structured approach for evaluating the ICT contribution to development.," International Journal on Advances in ICT for Emerging Regions (ICTer). , no. 7. 10.4038/ic, 2014.

[12] R. \&. M. A. Heeks, "Impact Assessment of ICT-for-Development Projects: A Compendium of Approaches.," no. 10.13140/RG.2.2.13011.53282., 2009.

[13] S. \&. K. K. Kozlowski, "A multilevel approach to theory and research in organizations: Contextual, temporal, and emergent processes. Multilevel theory, research, and methods in organizations: Foundations, extensions, and new directions," 2012.

[14] J. \&. A. H. \&. B. J. McGrath, "The Study of Groups: Past, Present, and Future. Personality and Social Psychology Review.," no. 4. 95-105. 10.1207/S15327957PSPR0401_8., 2000. 


\title{
International Journal of Advanced Research in Computer and Communication Engineering
}

\author{
Vol. 9, Issue 8, August 2020
}

\section{DOI 10.17148/IJARCCE.2020.9801}

[15] F.-F. Rothe:, "Rethinking positive and negative impacts of 'ICT for development' through the holistic lens of the sustainable development goals, Information Technology for Development.," no. 10.1080/02681102.2020.1756728, 2020.

[16] C. \&. F. T. May, "Implementing, embedding, and integrating practices: An outline of normalization process theory. Sociology.," pp. 43(3), 535554., 2009.

[17] R. B. L. M. S. O. C. A. M. F. S. \&. M. A. McEvoy, "A qualitative systematic review of studies using the normalization process theory to research implementation processes.," Implementation Science, Vols. 9(1), 2., 2015.

[18] E. M. C. \&. M. F. Murray, "Development and formative evaluation of the e-Health Implementation Toolkit (e-HIT).," BMC Medical Informatics and Decision Making., Vols. 10(1), , p. 61., 2010.

[19] J. I. K. E. K. T. O. H. T. R. R. \&. B. J. Saebo, "Comparing strategies to integrate health information systems following a data warehouse approach in four countries.," Information Technology for Development, , Vols. 17(1),, pp. 42-60, 2011.

[20] H. Misra, "Relevance of Measurements in E-Governance: Software Engineering Perspectives in Indian Context.," no. 10.13140/RG.2.1.4930.9282., 2015.

[21] L. G. F. M. \&. C. A. K. Tornatzky, "Processes of technological innovation.," Lexington books., 1990

[22] S. Bakry, "Development of e-government: a STOPE view. Int. Journal of Network Management.," no. 14. 339-350. 10.1002/nem., p. $529 ., 2004$.

[23] K. \&. A. \&. B. S. Al-osaimi, " An Integrated STOPE Framework for e-Readiness Assessment.," $2006 .$.

[24] S. \&. A. N. \&. D. G. Kamel, "A Suggested Framework for Assessing Electronic Government Readiness in Egypt.," Electronic Journal of eGovernment. , vol. 7, pp. 11-28, 2009.

[25] A. \&. E. S. \&. N. A. \&. A. T. \&. O. O. \&. A. D. Taiwo, "Health information technology in Nigeria: Stakeholders' perspectives of nationwide implementations and meaningful use of the emerging technology in the most populous black nation.," American Journal of Health Research., vol. 3. , no. 10.11648/j.ajhr. s.2015030101.13., pp. 17-24. , 2014.

[26] B. \&. G. I. Baridam, "ICT Influence on the healthcare sector in the Niger Delta region: ICT policy at an organizational level.," Development Studies Research., vol. 6., no. 142-151. 10.1080/21665095.2019.1680302., p. 2019.

[27] A. Obotu, "Perceived effect of telemedicine on medical service delivery by federal medical centers in north central zone of Nigeria.," 2019.

[28] K. O. P. a. S. A. James, "Telemedicine Development in Nigeria.," National Space Research and Development Agency (NASRDA), Abuja Nigeria, pp. 92-103., 2015.

[29] A. a. I. A. Matawalli, "Health Care Using Telemedicine: A Case Study of Yobe State, Nigeria.," International Journal of Computer Science and Network., no. 3, pp. 5 -11, 2014.

[30] D. \&. T. M. Blumenthal, "The 'Meaningful Use' Regulation for electronic health records," The New England Journal of Medicine., no. 363(6), 501-504. doi: 10.1056/NEJMp1006114, 2010.

[31] R. R. \&. D. S. D. Robey, "A tutorial on conducting meta-analyses of clinical outcome research.," Journal of Speech, Language, and Hearing Research, no. 41, 1227-1241., 1998.

[32] S. M. Liehr P, "Middle range theory: Spinning research and practice to create knowledge for the new millennium. Advances in Nursing Science.Advances in Nursing Science," no. 21(4):, pp. 81-91, 1999.

[33] K. M. Chinn PL, " Theory and Nursing: A Systematic Approach.," , no. 5th Edition. St Louis, USA:Mosby., 1999. 